CERN-TH/96-21

hep-ph/9602208

\title{
The Top Cross Section in HADRONIC COLLisions
}

\author{
Stefano CATANI" \\ INFN, Sezione di Firenze and Univ. di Firenze, Florence, Italy \\ Michelangelo L. MANGANO\{, Paolo NASON円, \\ CERN, TH Division, Geneva, Switzerland \\ Luca TRENTADUE ${ }^{1}$ \\ Univ. di Parma and INFN, Gruppo Collegato di Parma, Parma, Italy
}

\begin{abstract}
We reexamine the top quark production cross section at the Tevatron and LHC, in the light of recent progress on the resummation of logarithmic soft gluon corrections. We find that resummation effects are much smaller than previously thought. We also compute Coulombic threshold effects, and find them negligible. We update the discussion of uncertainties due to scale dependence, the value of the strong coupling constant, and the parton density parametrization. Our current best estimate of the top production cross section at the Tevatron and its error is $\sigma\left(m_{\mathrm{t}}=175 \mathrm{GeV}\right)=4.75_{-0.62}^{+0.73}$.
\end{abstract}

CERN-TH/96-21

September 4, 2018

\footnotetext{
${ }^{1}$ Research supported in part by the EEC programme "Human Capital and Mobility", Network "Physics at High Energy Colliders", contract CHRX-CT93-0357(DG 12 COMA).

${ }^{2}$ On leave of absence from INFN, Pisa, Italy

${ }^{3}$ On leave of absence from INFN, Milan, Italy
} 


\section{Introduction}

We present in this letter a theoretical reassessment of the evaluation of the top quark production cross section in high-energy hadronic collisions. The top quark having been found [1, 2], the comparison between its observed production properties and those expected from the Standard Model will be an important probe of the possible existence of new phenomena. One of the most important tests to be performed concerns the total production cross section. This is the most inclusive quantity available, and is a priori the least sensitive to a detailed understanding of the higher order corrections which influence the evolution of the initial and final states. Within QCD, one expects the perturbative expansion in powers of $\alpha_{s}\left(m_{\mathrm{t}}\right)$ to be well behaved and to provide an accurate estimate of the total cross section already at low orders. In particular, the first estimates of the total production cross section using the full next-to-leading-order (NLO) matrix elements [3, 4, 5] gave an increase (relative to the Born result) of the order of $30 \%$ for masses above $100 \mathrm{GeV}$. The residual perturbative QCD uncertainty, evaluated by varying the renormalization and factorization scales, was shown to be no larger than $10 \%$. The choice of parametrization for the input parton densities was also shown to give effects of this order of magnitude, by using the available sets.

It was pointed out in ref. [6] that logarithmic contributions associated to the emission of soft gluons from the initial state could significantly enhance the NLO result. An independent study of the soft gluon resummation in top production has appeared recently [7]. These studies are based on the resummation formulae obtained in the pioneering works of refs. [8, 9, 10]. Using these formulae for explicit calculations requires the choice of a prescription in order to bypass the Landau-pole singularity which is exposed by the integration of the QCD running coupling over gluon energies of the order of $\Lambda_{Q C D}$. In particular it was shown in ref. [6] that, within the proposed formalism, one can get sensible results only by cutting off the soft gluon emission at scales below $0.1 \div 0.2 m_{\mathrm{t}}$. The choice made in ref. [7] follows instead a suggestion put forward in ref. [11. According to this prescription the Landau pole appearing in the Mellin transform of the resummed hard cross section should be integrated over in a principal value sense. It was argued in ref. [11] that this prescription results in

non-perturbative power-suppressed ambiguities scaling like $\Lambda_{Q C D} / Q$ in the hard scale $Q$ typical of the process under consideration. The net effect of gluon resummation evaluated in ref. [7] amounts to an increase by approximately $10 \%$ of the NLO cross 
section.

In a companion paper [12] we will show that the approaches of refs. [6, 7] overestimate both the gluon resummation contribution and the associated non-perturbative residual uncertainty. This is a consequence of the fact that their resummation formulae introduce unjustified factorially growing terms in the perturbative expansion. In ref. 12 we will also show that a more natural prescription exists, in which these terms are not present. In the following, we will briefly summarize the basis of our criticism and the definition and main properties of the new proposed resummation prescription. We will then present a numerical study indicating that the impact of resummation on the total top production cross section is of the order of a percent, much smaller than previously thought. We will also show that threshold corrections due to higher order Coulomb effects are likewise negligible. We will conclude this study with an updated analysis of the current theoretical uncertainties coming from the scale dependence, from the choice of partonic densities and from the value of $\alpha_{s}$.

It should be pointed out that this study is performed within the strict domain of the Standard Model. Corrections to the top cross section much larger than the Standard Model QCD uncertainties can be obtained in the presence of new phenomena. For a partial list of specific examples, see e.g. refs. [13.

\section{The minimal prescription for the resummation}

The heavy flavour production cross section in the Born approximation is given by the formula

$$
\sigma=\sum_{i, j=q \bar{q}, \bar{q} q, g g} \int_{0}^{1} d x_{1} d x_{2} F^{(1, i)}\left(x_{1}\right) F^{(2, j)}\left(x_{2}\right) \hat{\sigma}^{(i j)}\left(\frac{\rho}{x_{1} x_{2}}\right), \quad \rho=\frac{4 m^{2}}{S},
$$

where $m$ is the mass of the heavy quark, and $S$ is the square of the total centre-ofmass energy. The functions $F^{(i, k)}$ are the parton densities for parton $k$ in hadron $i$, and are evaluated at a scale $\mu$ of the order of the heavy quark mass $m$. Explicit

formulae for the partonic cross section $\hat{\sigma}$ can be found for example in ref. [3]. The corresponding $N$ space formula is

$$
\sigma=\sum_{i, j=q \bar{q}, \bar{q} q, g g} \frac{1}{2 \pi i} \int_{C-i \infty}^{C+i \infty} F_{N+1}^{(1, i)} F_{N+1}^{(2, j)} \hat{\sigma}_{N}^{(i j)} \rho^{-N} d N
$$


where the Mellin transforms are defined in the following way

$$
F_{N}=\int_{0}^{1} \frac{d x}{x} x^{N} F(x), \quad \hat{\sigma}_{N}=\int_{0}^{1} \frac{d z}{z} z^{N} \hat{\sigma}(z) .
$$

The integration contour lies to the right of all singularities.

We use the following resummed formula

$$
\sigma^{(\mathrm{res})}=\sum_{i, j=q \bar{q}, \bar{q} q, g g} \frac{1}{2 \pi i} \int_{C_{\mathrm{MP}}-i \infty}^{C_{\mathrm{MP}}+i \infty} F_{N+1}^{(1, i)} F_{N+1}^{(2, j)} \Delta_{N+1}^{(i j)} \hat{\sigma}_{N}^{(i j)} \rho^{-N} d N .
$$

where, in the $\overline{\mathrm{MS}}$ scheme

$$
\ln \Delta_{N}^{(i j)}=\ln N g_{i j, 1}\left(b_{0} \alpha_{s} \ln N\right)+\mathcal{O}\left(\alpha_{s}^{k} \ln ^{k} N\right)
$$

where

$$
\begin{aligned}
& g_{q \bar{q}, 1}(\lambda)=g_{1}^{\overline{\mathrm{MS}}}(\lambda), \quad g_{g g, 1}(\lambda)=\frac{C_{A}}{C_{F}} g_{1}^{\overline{\mathrm{MS}}}(\lambda) \\
& g_{1}^{\overline{\mathrm{MS}}}(\lambda)=+\frac{C_{\mathrm{F}}}{\pi b_{0} \lambda}[2 \lambda+(1-2 \lambda) \ln (1-2 \lambda)],
\end{aligned}
$$

and

$$
b_{0}=\frac{11 C_{A}-2 n_{f}}{12 \pi} \quad C_{A}=3, \quad C_{F}=\frac{4}{3} .
$$

In formula (2.5) the strong coupling constant $\alpha_{s}$ is evaluated at a scale $\mu$ of the order of the heavy quark mass $m$. The factor $\ln \Delta_{N}^{(i j)}$ resums all the leading logarithmic terms $\alpha_{s}^{k} \ln ^{k+1} N$ due to soft gluon emission. The contour in eq. (2.4) is chosen between the cuts on the negative $N$ axis and the singularity at $b_{0} \alpha_{s} \log N=1 / 2$, which is a Landau pole. We will call this the minimal prescription (MP). In ref. [12] we will show that this prescription enjoys the following remarkable properties:

- It is accurate at the leading log level in the threshold limit.

- If we expand the MP formula in powers of $\alpha(m)$, the expansion is asymptotic to the full formula.

- The power expansion of the MP formula does not have any factorially growing coefficients, and therefore is free of ambiguities of the order of powers of $\Lambda / \mathrm{m}$. The ambiguity associated with the asymptotic expansion of the MP resummation is exponentially suppressed, being of the order $e^{-B(1-\rho) \frac{m}{\Lambda}}$. 
We claim that previous calculations of resummation effects [6, 7] have a corresponding power expansion which does have factorially growing terms. These terms are spurious. They arise either from certain approximations performed when going from the $N$ space to the $x$ space formulae, or from the integration of the running coupling constant down to the Landau singularity.

Our result is consistent with the findings of ref. [14], indicating that no power-like $1 / Q$ ambiguity emerges from the resummation of multiple gluon emission in Drell-Yan processes.

A full discussion of these issues will appear in a companion paper [12, where we will also study the implications of this prescription for Drell-Yan, heavy quark and jet production in hadronic collisions.

In the present work we will report on results obtained with our MP formula of eq. (2.4), which was implemented without any further approximation in a numerical program.

\section{Results}

In this section we present the results obtained for a very heavy quark, and in particular for the top cross section. First of all, we remind the reader that large soft gluon effects are present not only in the production cross section, but also in the deep-inelastic processes that are used to determine the structure functions. In order to perform a complete study of the resummation one should therefore use resummed formulae also when fitting deep-inelastic scattering data, direct photon production data, and in general all phenomena which are used to constrain the parton densities. In the present work we will assume that the structure functions have been properly extracted from data (including the resummation effects) and assess the significance of the resummation for heavy flavour production. Effects of comparable size may arise from refitting the structure functions using resummed formulae. Our conclusions will mostly be based upon the fact that the resummation effects we find are in fact very small, and can be neglected.

The importance of the resummation effects is illustrated in fig. 1 where we plot the quantities

$$
\frac{\delta_{\mathrm{gg}}}{\sigma_{\mathrm{NLO}}^{(g g)}}, \quad \frac{\delta_{\mathrm{q} \overline{\mathrm{q}}}}{\sigma_{\mathrm{NLO}}^{(q \bar{q})}}, \quad \frac{\delta_{\mathrm{gg}}+\delta_{\mathrm{q} \overline{\mathrm{q}}}}{\sigma_{\mathrm{NLO}}^{(g g)}+\sigma_{\mathrm{NLO}}^{(q \bar{q})}}
$$




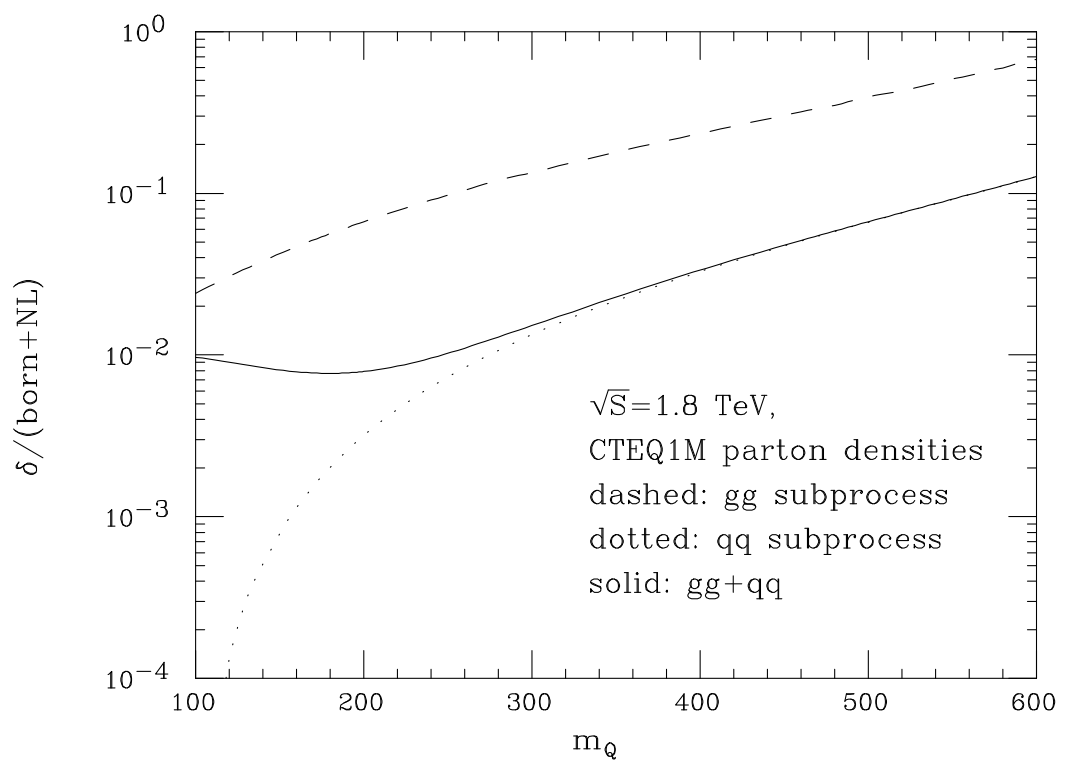

Figure 1: Contribution of gluon resummation at order $\alpha_{s}^{4}$ and higher, relative to the NLO result, for the individual subprocesses and for the total, as a

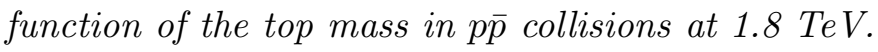

where $\delta$ is equal to our MP resummed hadronic cross section (2.4) in which the terms of order $\alpha_{s}^{2}$ and $\alpha_{s}^{3}$ have been subtracted, and $\sigma_{(\mathrm{NLO})}$ is the full hadronic NLO cross section [3]. In other words, what we display is the relative contribution to the total cross section coming from corrections of order higher than those accounted for by the full NLO result. We show the results for the $q \bar{q}$ and $g g$ production channels separately, as well as for the total. The results are shown at $\sqrt{S}=1.8 \mathrm{TeV}$ as a function of the top mass in the range $100<m_{\mathrm{t}}(\mathrm{GeV})<600$. The wide mass range is chosen for the sole purpose of illustration. We chose a common renormalization and factorization scale $\mu=m_{\mathrm{t}}$, and parton densities from the CTEQ1 set [15.

The plot clearly indicates that the contribution of soft-gluon resummation is negligible, unless the top mass approaches the total hadronic energy. This result is consistent with the expectation that the soft gluon enhancement of the production cross sections should be relevant only very close to threshold. For a top mass of $175 \mathrm{GeV}$ the increase due to this effect is below $1 \%$. We also note that the effect of resummation is potentially much larger for the $g g$ channel, because of the $C_{\mathrm{A}} / C_{\mathrm{F}}$ enhancement in the exponent of the resummation formula (see eq. 2.6). It turns out, however, that when the $g g$ subprocess becomes important (i.e. for small top masses) 


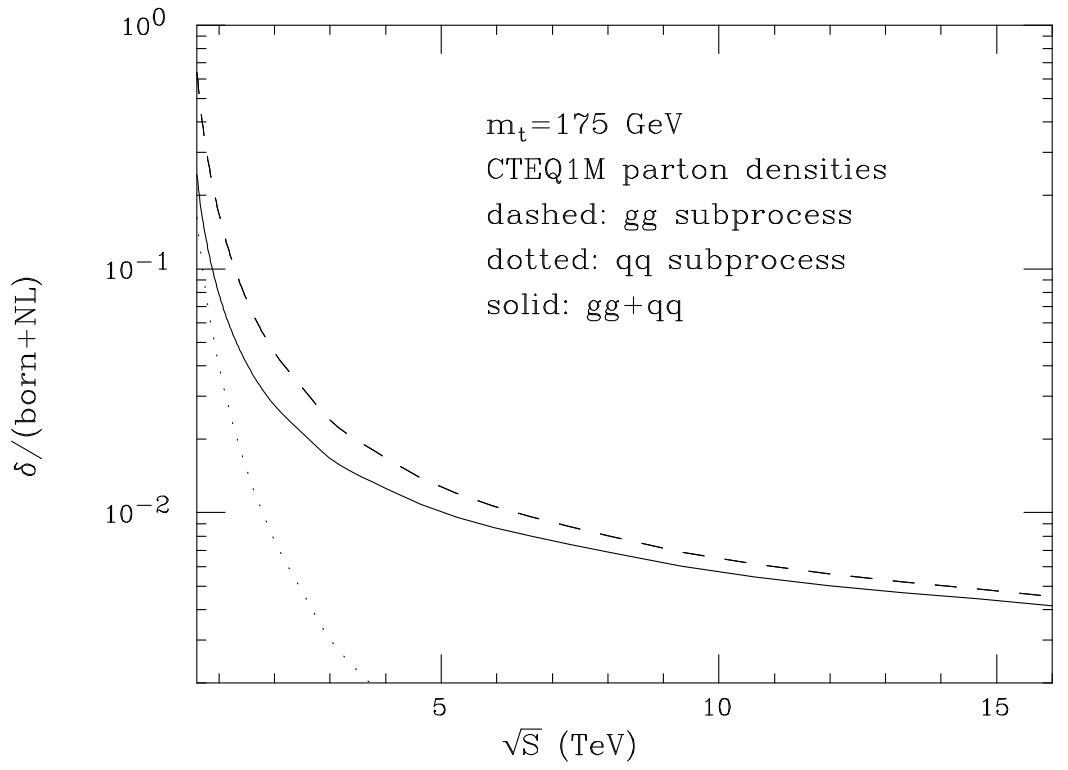

Figure 2: Contribution of gluon resummation at order $\alpha_{s}^{4}$ and higher, relative to the NLO result, for the individual channels and for the total, as a function of the CM energy in pp collisions.

we are so far from threshold that the resummation effect is very small. Conversely, for large masses, the quark component, which has smaller resummation effects, dominates.

In fig. 2 we show the same quantities, at fixed top mass, versus the CM energy. We see that at the LHC, where production is dominated by the $g g$ initial state, we are far enough from the hadronic threshold, so that the resummation effects are again small. We conclude therefore that the effects of gluon resummation can be neglected at the present stage, as they are much smaller than the uncertainties present in the NLO determination. Note that, due to the dominance of the $g g$ subprocess, the relative importance of the resummation effects at $\sqrt{S}=1.8 \mathrm{TeV}$ is larger in $p p$ collisions than in $p \bar{p}$.

Beside the soft gluon emission effects, also Coulomb effects may enhance or deplete the cross section near threshold [16, 17]. We have calculated these effects in the following way. We have separated the partonic Born cross section formulae into their colour singlet and octet components

$$
\hat{\sigma}^{(g g)}=\hat{\sigma}_{(8)}^{(g g)}+\hat{\sigma}_{(1)}^{(g g)}
$$




$$
\hat{\sigma}^{(q \bar{q})}=\hat{\sigma}_{(8)}^{(q \bar{q})}
$$

where $\hat{\sigma}^{(q \bar{q}, g g)}$ can be found in refs. [3], and

$$
\hat{\sigma}_{(1)}^{(g g)}(\rho)=\frac{\alpha_{s}^{2}}{m^{2}} \frac{\beta \rho \pi}{384}\left[\frac{1}{\beta} \log \frac{1+\beta}{1-\beta}\left(4+4 \rho-2 \rho^{2}\right)-4-4 \rho\right],
$$

where $\beta=\sqrt{1-\rho}$. The Coulomb-resummed cross section is given as

$$
\hat{\sigma}^{\text {Coul }}(\rho)=\hat{\sigma}_{(8)}(\rho) \frac{\pi \alpha_{s} /(6 \beta)}{\exp \left(\pi \alpha_{s} /(6 \beta)\right)-1}+\hat{\sigma}_{(1)}(\rho) \frac{4 \pi \alpha_{s} /(3 \beta)}{1-\exp \left(-4 \pi \alpha_{s} /(3 \beta)\right)} .
$$

We do not include bound state effects, which, as shown in ref. [17], are much smaller. In fig. 3 we have plotted the hadronic quantity

$$
\frac{\delta_{\mathrm{Coul}}}{\sigma_{(\mathrm{NLO})}}
$$

which (similarly to the case of soft gluon effects) represents the relative correction due to the resummation of Coulomb effects not already included into the NLO results. We see that these effects are similar in magnitude to the soft-gluon effects, and fully negligible at the Tevatron. Since at the LHC we are further away from the threshold region, we conclude that also there they will be negligible. Therefore, the following estimates of the top cross section will not include neither soft gluon effects, nor Coulombic ones. Electroweak corrections to top production in hadronic collisions have been considered in refs. [18]. They range from $-0.97 \%$ to $-1.74 \%$ of the born cross section, for a higgs mass of 60 and $1000 \mathrm{GeV}$ respectively [19. They will also not be included in the following estimates.

We now proceed to review the current theoretical uncertainties that arise at NLO. Uncertainties due to unknown higher-order effects are usually accounted for by varying the renormalization $\left(\mu_{\mathrm{R}}\right)$ and factorization $\left(\mu_{\mathrm{F}}\right)$ scales. In principle, independent variations of the two scales should be considered. In fig. Đ we show the scale dependence of the top cross section. Note that there is compensation of the factorization scale dependence when the different subprocesses are added up. In particular, the $g g$ and $q g$ factorization scale dependence have opposite behaviour. In fact, it is only the combined scale dependence that can be considered an estimate of the neglected subleading corrections. As a second point, we observe that the maximum of the cross section is reached around $m_{t} / 2$. Thus, the usual choice of the range $m_{t} / 2<\mu<2 m_{t}$ appears to be particularly justified in this case. As a third point, we observe that the 


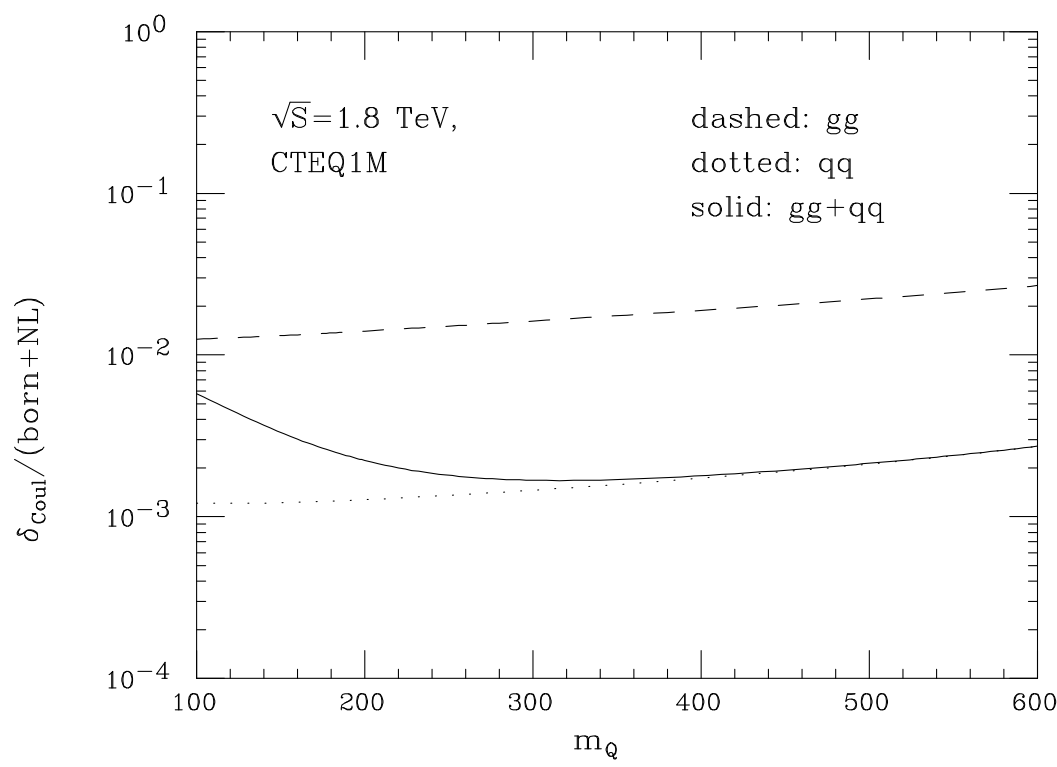

Figure 3: Contribution of Coulomb effects at order $\alpha_{s}^{4}$ and higher, relative to the NLO result, for the individual channels and for the total, as a function of the CM energy in p $\bar{p}$ collisions.

scale dependence in the cross section goes in the same direction for the two scales, so that, for the purpose of estimating the associated uncertainty, it is sufficient to consider the simultaneous variation of $\mu_{\mathrm{R}}$ and $\mu_{\mathrm{F}}$.

Aside from the scale uncertainties, which reflect the limitations of the perturbative QCD calculation, there are uncertainties associated to our imprecise knowledge of the physical parameters involved. In particular, the strong coupling constant is determined within a certain accuracy. In the case of top production at the Tevatron, larger strong couplings tend to give larger partonic cross section. However, for larger strong couplings, the Altarelli-Parisi evolution, which occurs along the wide span in $Q^{2}$ from the values at which deep-inelastic fits are performed $\left(\approx 10 \mathrm{GeV}^{2}\right)$ to the top mass, softens the quark parton densities, thereby decreasing the cross section. In order to perform a fair estimate of the uncertainty due to $\Lambda_{\mathrm{QCD}}$, we need sets of parton densities fitted with different values of the strong coupling. The sets of ref. 20] meet our purposeft. In fig. 5 we show the cross section as a function of the strong coupling.

\footnotetext{
${ }^{4}$ The values of $\Lambda_{4}$ that accompany the fortran program for the structure function sets of ref. [20] are not consistent with the values of $\alpha_{s}$ quoted there, the differences being of the order of $1 \%$. In the present work, we extract the values of $\Lambda_{5}$ from their quoted values of $\alpha_{s}$ using the standard
} 


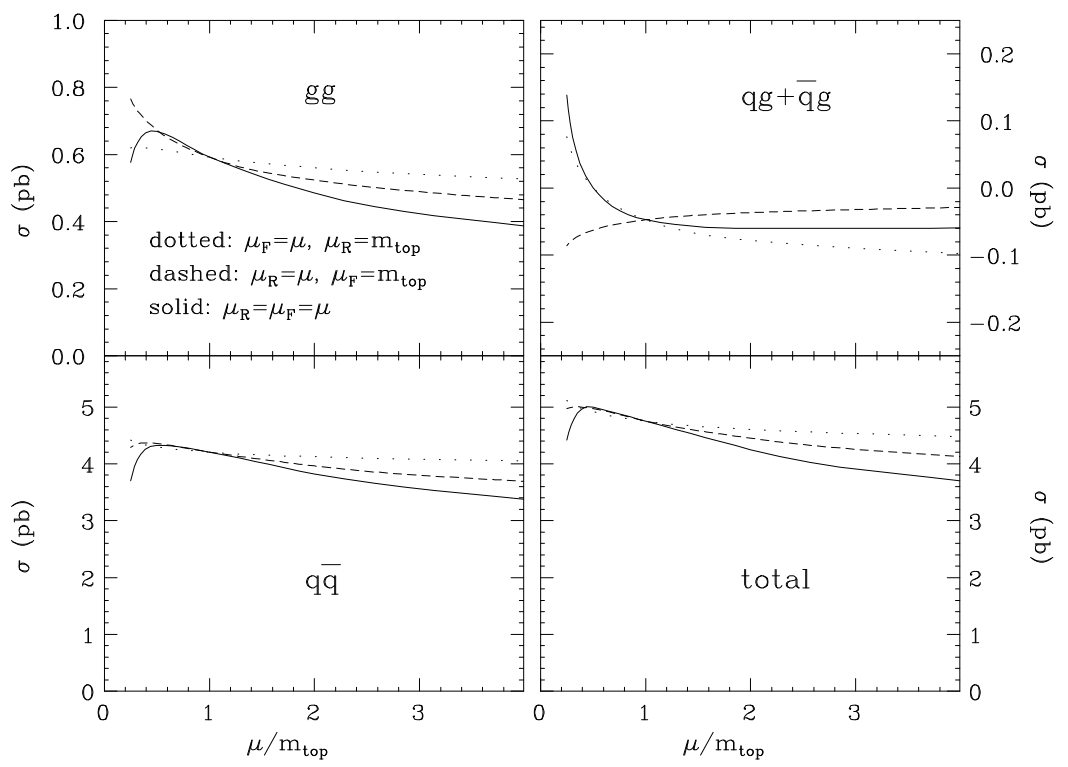

Figure 4: Scale dependence of the top cross section at the Tevatron. Dotted lines are the $\mu_{\mathrm{F}}$ dependence at fixed $\mu_{\mathrm{R}}$, dashed lines are the $\mu_{\mathrm{R}}$ dependence at fixed $\mu_{\mathrm{F}}$, and solid lines are obtained by the simultaneous variation of $\mu_{\mathrm{R}}$ and $\mu_{\mathrm{F}}$. 


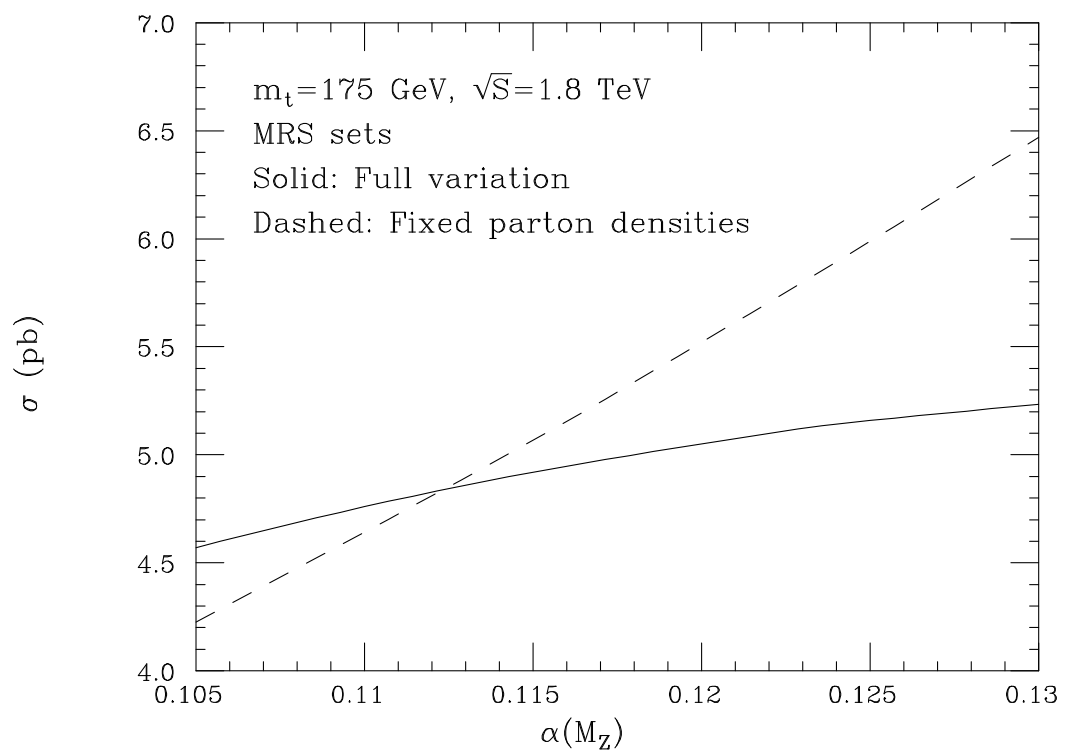

Figure 5: Top cross section as a function of $\alpha_{s}\left(M_{\mathrm{Z}}\right)$. The dotted line does not include the variation of the parton densities due to the change in $\alpha_{s}$.

For comparison we also show the $\alpha_{s}$ dependence if the parton densities are kept fixed. We see the remarkable reduction in the $\alpha_{s}$ dependence due to the compensation of the rise of the partonic cross section and the decrease of the quark parton densities.

Our results for the top cross section are collected in tables 1, 2 and 3, for values of the top mass that span the current uncertainties. We also show results obtained with with the recent parametrization derived in ref. [22 by including the CDF jet data. We stress that the numbers in the table are obtained with a standard two-loop calculation. No resummation effects are included.

Our ranges for the top cross section are thus given by $7.78_{-1.06}^{+1.28} \mathrm{pb}, 4.75_{-0.62}^{+0.73} \mathrm{pb}$, $2.97_{-0.38}^{+0.42} \mathrm{pb}$ for $m_{\mathrm{t}}=160,175,190 \mathrm{GeV}$ respectively, and are adequately fitted in this range by the expression

$$
\sigma(t \bar{t})=e^{\frac{175-m_{\mathrm{t}}}{31.5}}\left(4.75_{-0.62}^{+0.73}\right) .
$$

As a central value for our determination we have chosen the MRSA' 23] result with $\mu_{\mathrm{R}}=\mu_{\mathrm{F}}=m_{\mathrm{t}}$, in association with a value of $\Lambda_{5}=0.152 \mathrm{GeV}$ (which corresponds to $\alpha_{s}\left(M_{Z}\right)=0.1113$, according to the standard two-loop formula [21]). For the MRS sets with variable $\Lambda$, we have used $\Lambda_{5}=0.0994, .140, .190, .253, .328 \mathrm{GeV}$ (which two-loop formula 21. 


\begin{tabular}{|c|c|c|c|c|c|c|c|c|}
\hline & \multirow{2}{*}{$\mu_{\mathrm{R}}=\mu_{\mathrm{F}}$} & & & & \multirow{2}{*}{ CTEQ1M $^{*}$} & CTEQ $^{\prime}$ & MRSA $^{\prime}$ & \multicolumn{5}{|c|}{ MRS, variable $\Lambda, \alpha_{s}\left(M_{\mathrm{Z}}\right)=$} \\
\cline { 5 - 9 } & & & & 0.105 & 0.110 & 0.115 & 0.120 & 0.125 \\
\hline \hline$m_{\mathrm{t}} / 2$ & 5.24 & 5.07 & 5.00 & 4.78 & 4.99 & 5.18 & 5.34 & 5.48 \\
\hline$m_{\mathrm{t}}$ & 4.96 & 4.86 & 4.75 & 4.57 & 4.76 & 4.92 & 5.05 & 5.16 \\
\hline $2 m_{\mathrm{t}}$ & 4.38 & 4.38 & 4.25 & 4.13 & 4.27 & 4.38 & 4.47 & 4.52 \\
\hline
\end{tabular}

Table 1: Total cross sections for $m_{\mathrm{t}}=175$ GeV at NLO.

\begin{tabular}{|c|c|c|c|c|c|c|c|c|}
\hline & \multirow{2}{*}{$\mu_{\mathrm{R}}=\mu_{\mathrm{F}}$} & & & \multirow{2}{*}{ CTEQ1M } & CTEQ $^{\prime}$ & MRSA $^{\prime}$ & \multicolumn{5}{|c|}{ MRS, variable $\Lambda, \alpha_{s}\left(M_{\mathrm{Z}}\right)=$} \\
\cline { 5 - 9 } & & & & 0.105 & 0.110 & 0.115 & 0.120 & 0.125 \\
\hline \hline$m_{\mathrm{t}} / 2$ & 8.64 & 8.18 & 8.19 & 7.80 & 8.17 & 8.50 & 8.81 & 9.06 \\
\hline$m_{\mathrm{t}}$ & 8.16 & 7.85 & 7.78 & 7.45 & 7.78 & 8.06 & 8.32 & 8.53 \\
\hline $2 m_{\mathrm{t}}$ & 7.21 & 7.08 & 6.95 & 6.72 & 6.97 & 7.18 & 7.35 & 7.48 \\
\hline
\end{tabular}

Table 2: Total cross sections for $m_{\mathrm{t}}=160 \mathrm{GeV}$ at $N L O$. 


\begin{tabular}{|c|c|c|c|c|c|c|c|c|}
\hline \hline \multirow{2}{*}{$\mu_{\mathrm{R}}=\mu_{\mathrm{F}}$} & \multirow{2}{*}{ CTEQ1M } & \multirow{2}{*}{ CTEQ $^{\prime}$} & \multirow{2}{*}{ MRSA $^{\prime}$} & \multicolumn{5}{|c|}{$\mathrm{MRS}$, variable $\Lambda, \alpha_{s}\left(M_{\mathrm{Z}}\right)=$} \\
\cline { 5 - 9 } & & & & 0.105 & 0.110 & 0.115 & 0.120 & 0.125 \\
\hline \hline$m_{\mathrm{t}} / 2$ & 3.26 & 3.22 & 3.13 & 3.00 & 3.13 & 3.24 & 3.32 & 3.39 \\
\hline$m_{\mathrm{t}}$ & 3.08 & 3.08 & 2.97 & 2.87 & 2.98 & 3.07 & 3.14 & 3.20 \\
\hline $2 m_{\mathrm{t}}$ & 2.72 & 2.77 & 2.66 & 2.59 & 2.67 & 2.73 & 2.78 & 2.78 \\
\hline
\end{tabular}

Table 3: Total cross sections for $m_{\mathrm{t}}=190$ GeV at NLO.

corresponds to $\left.\alpha_{s}\left(M_{Z}\right)=0.105,0.110,0.115,0.120,0.125\right)$.

In $p \bar{p}$ collisions at $\sqrt{S}=2 \mathrm{TeV}$ we obtain, with the same method, a top cross section of $10.5_{-1.4}^{+1.8} \mathrm{pb}, 6.53_{-0.86}^{+1.03} \mathrm{pb}, 4.17_{-0.53}^{+0.61} \mathrm{pb}$ for $m_{\mathrm{t}}=160,175,190 \mathrm{GeV}$ respectively. The cross section bands are also shown in fig. 6, for $\sqrt{S}=1.8 \mathrm{TeV}$ and fig. 7 for $\sqrt{S}=2 \mathrm{TeV}$. Because of the high precision of this theoretical prediction, the measurement of the top cross section could become a sensitive probe of new physics 13. 24.

For reference, we also quote the cross section for top production at the LHC. We get $\sigma(t \bar{t})=0.77_{-0.12}^{+0.25} \mathrm{nb}$, for $m_{t}=175 \mathrm{GeV}$ and $\sqrt{S}=14 \mathrm{TeV}$. The error is obtained with the same scale and $\alpha_{s}$ variations we used for the Tevatron cross section.

\section{Acknowledgements}

We wish to thank R.K. Ellis for useful comments. We also wish to thank W. Hollik and D. Wackeroth for computing the value of the electroweak corrections to top production quoted above. Two of us (SC and LT) acknowledge the hospitality and the partial support received by the CERN TH division while this work was carried out. 


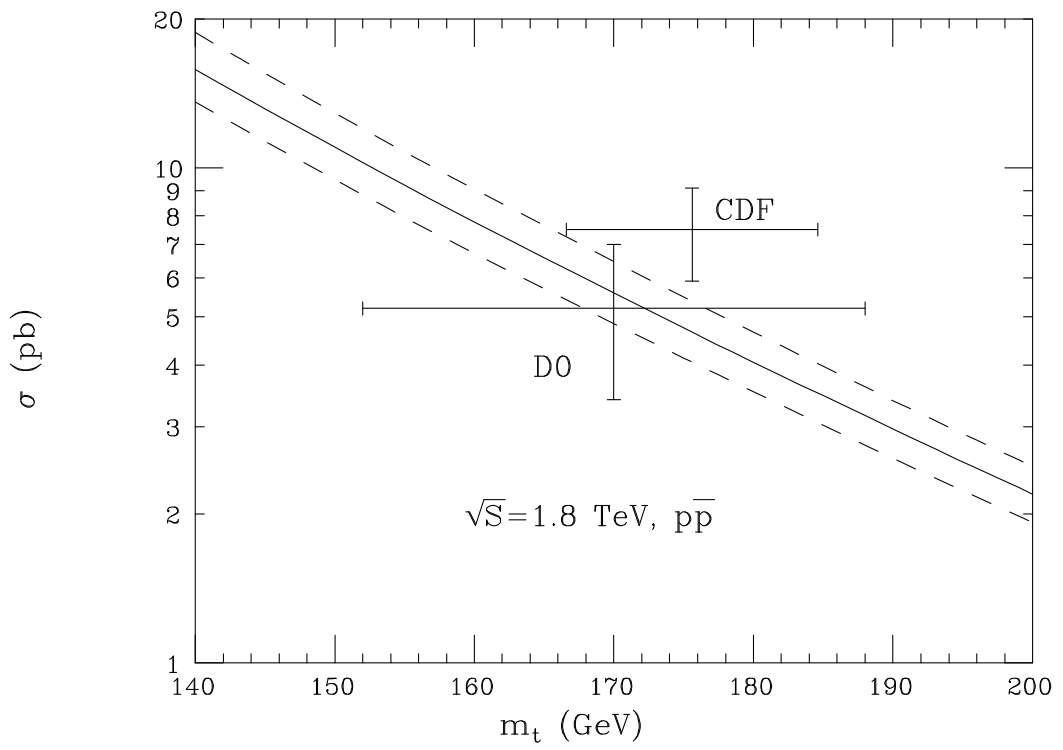

Figure 6: Top cross section at the Tevatron at $\sqrt{S}=1.8 \mathrm{TeV}$. The solid line is obtained with MRSA' parton density, and the dashed lines correspond to the upper and lower values obtained in tables 1-3. The experimental data are taken from ref. 205].

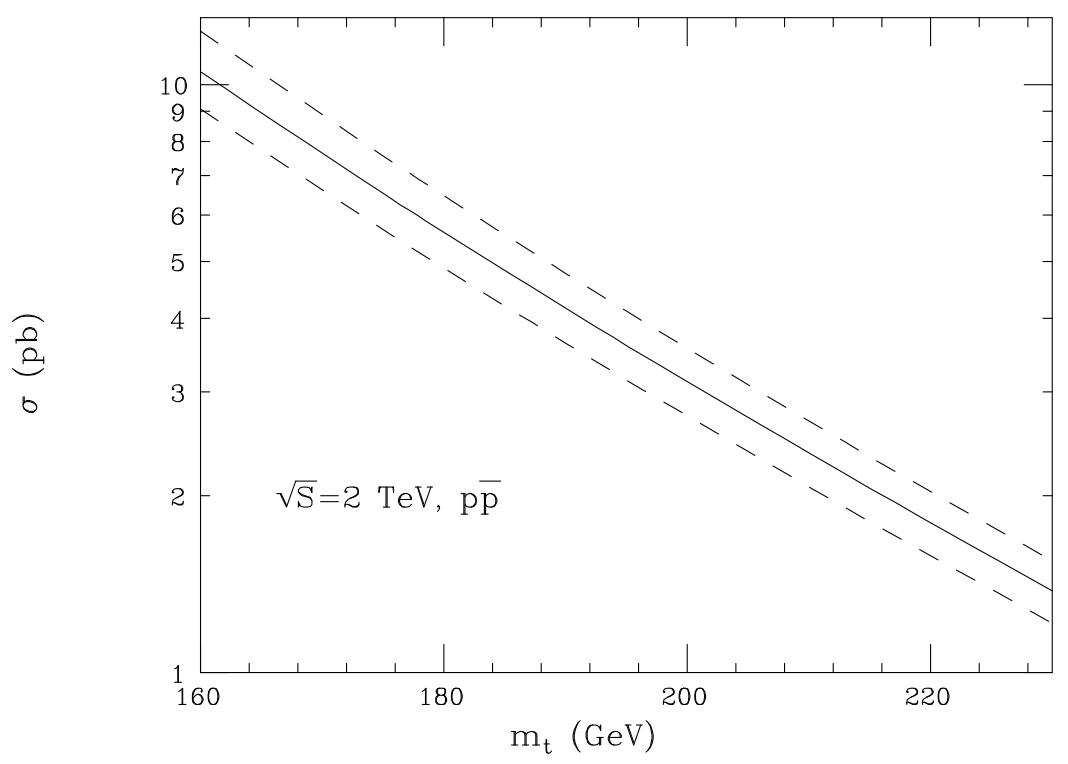

Figure 7: Top cross section at the Tevatron at $\sqrt{S}=2$ TeV. 


\section{References}

[1] F. Abe et al., CDF Collab., Phys. Rev. Lett. 74 (1995) 2626;

F. Abe et al., CDF Collab., Phys. Rev. D50 (1994) 2966.

[2] S. Abachi et al., D0 Collab., Phys. Rev. Lett. 74 (1995) 2632.

[3] P. Nason, S. Dawson and R. K. Ellis, Nucl. Phys. B303 (1988) 607;

W. Beenakker, H. Kuijf, W.L. van Neerven and J. Smith, Phys. Rev. D40 (1989) 54.

[4] G. Altarelli, M. Diemoz, G. Martinelli and P. Nason, Nucl. Phys. B308 (1988) 724 .

[5] R.K. Ellis, Phys. Lett. 259B (1991) 492.

[6] E. Laenen, J. Smith and W.L. van Neerven, Nucl. Phys. B369 (1992) 543; Phys. Lett. 321B (1994) 254.

[7] E. Berger and H. Contopanagos, Phys. Lett. 361B (1995) 115;

preprint ANL-HEP-CP-95-85, hep-ph/9512212.

[8] Yu.L. Dokshitzer, D.I. Dyakonov and S.I. Troyan, Phys. Rep. 58 (1980) 271;

G. Parisi, Phys. Lett. 90B (1980) 295;

G. Curci and M. Greco, Phys. Lett. 92B (1980) 175;

D. Amati et al., Nucl. Phys. B173 (1980) 429;

M. Ciafaloni and G. Curci, Phys. Lett. 102B (1981) 352;

P. Chiappetta, T. Grandou, M. Le Bellac and J.L. Meunier, Nucl. Phys. B207 (1982) 251.

[9] G. Sterman, Nucl. Phys. B281 (1987) 310.

[10] S. Catani and L. Trentadue, Nucl. Phys. B327 (1989) 323;

Nucl. Phys. B353 (1991) 183.

[11] G. Sterman and H. Contopanagos, Nucl. Phys. B419 (1994) 77;

L. Alvero and H. Contopanagos, Nucl. Phys. B436 (1995) 184.

[12] S. Catani, M.L. Mangano, P. Nason and L. Trentadue, in preparation. 
[13] E. Eichten and K. Lane, Phys. Lett. 327B (1994) 129;

C.T. Hill and S.J. Parke, Phys. Rev. D49 (1994) 4454;

R. Casalbuoni et al., CPT-95/P.3176, UGVA-DPT 1995/04-887, hepph/9505212;

B. Holdom, M.V. Ramana, Phys. Lett. 353B (1995) 295.

[14] M. Beneke and V.M. Braun, Nucl. Phys. B454 (1995) 253;

Yu.L. Dokshitser, G. Marchesini and B.R. Webber, preprint CERN-TH-95-281, hep-ph/9512336.

[15] J. Botts et al., Phys. Lett. 304B (1993) 159.

[16] A. Sommerfeld "Atombau und Spektrallinien", Bd. 2 (Vieweg, Braunschweig, 1939); A.D. Sakharov, JETP 18 (1948) 631.

[17] V. Fadin, V. Khoze and T. Sjöstrand, Z. Phys. C48 (1990) 613.

[18] S. Willenbrock and A. Stange, Phys. Rev. D48 (1993) 2054;

W. Beenakker, A. Denner, W. Hollik, R. Mertig, T. Sack and D. Wackeroth, Nucl. Phys. B411 (1994) 343;

C. Kao, G.A. Ladinsky, C.P. Yuan FSU-HEP-930508, hep-ph/9305270;

R. Harlander, M. Jezabeck and J.H. Kühn, TTP 95-25, hep-ph/9506292.

[19] W. Hollik and D. Wackeroth, private communication.

[20] A.D. Martin, R.G. Roberts and W.J. Stirling, Phys. Lett. 356B (1995) 89.

[21] L. Montanet et al., Phys. Rev. D50 (1994) 1173 and 1995 off-year partial update for the 1996 edition available on the PDG WWW pages (URL: http://pdg.lbl.gov/).

[22] J. Huston et al., Michigan State Preprint MSU-HEP-50812, hep-ph/9511386.

[23] A.D. Martin, R.G. Roberts and W.J. Stirling, Phys. Rev. D50 (1994) 6734.

[24] J.M. Yang and C.S. Li, Phys. Rev. D52 (1995) 1541;

C.S. Li, B.Q. Hu, J.M. Yang and C.G. Hu, Phys. Rev. D52 (1995) 1541.

[25] A. Caner, for the CDF Collaboration, presented at the $10^{\text {th }}$ Les Rencontres de Physique de la Vallée d'Aoste, La Thuile, Val d'Aosta, March 3-9, 1996; 
M. Narain, for the D0 Collabration, presented at the $10^{\text {th }}$ Les Rencontres de Physique de la Vallée d'Aoste, La Thuile, Val d'Aosta, March 3-9, 1996. 